\title{
Formation of Gold Nanoparticles in Water-in-Oil Microemulsions: Experiment, Mechanism, and
}

\section{Simulation}

\author{
Anil Rajapantulu, Rajdip Bandyopadhyaya* \\ Department of Chemical Engineering, Indian Institute of Technology Bombay, Powai, \\ Mumbai 400076, India
}

*Corresponding author

Email: rajdip@che.iitb.ac.in

Fax: +91(22) 25726895

\section{CONTENTS}

1. Morphology of nanoparticles at high water to surfactant ratio.

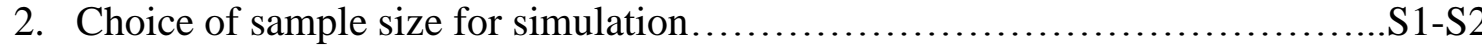

3. Different probable coagulation mechanisms...............................S3

4. Comparison of particle size distribution from experiment and simulation.......S4-S5

5. Choice of nature of drop to be added after coagulation of two drops, each containing a particle $. \mathrm{S} 5-\mathrm{S} 6$

6. References. 


\section{Morphology of nanoparticles at high water to surfactant ratio:}

At high water to surfactant ratio $(R)$, interconnected cluster of nanoparticle was observed as, shown in Figure S1.

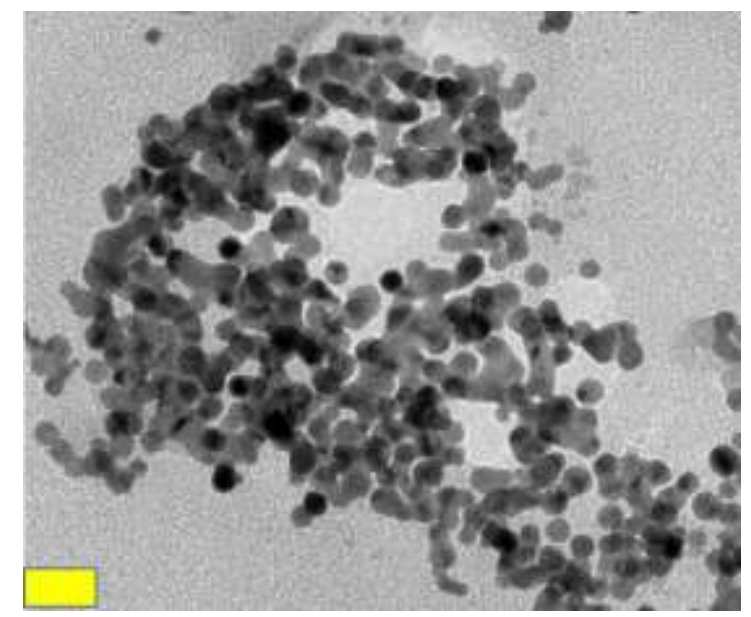

Figure S1. TEM image of GNPs at, $\mathrm{t}=5 \mathrm{~min}, R=10$. GNPs synthesized with $\left[\mathrm{HAuCl}_{4}\right]=1 \mathrm{mM}$, $\left[\mathrm{NaBH}_{4}\right]=2 \mathrm{mM}$, and $[$ AOT $]=0.1 \mathrm{M}$. Scale bar represents $20 \mathrm{~nm}$.

\section{Choice of sample size for simulation:}

In order to see the effect of sample size on our simulation results, we varied our drop population $\left(\mathrm{N}_{\mathrm{d}}\right)$, shown in Figure S2. Clearly, the particle mean diameter and standard deviation

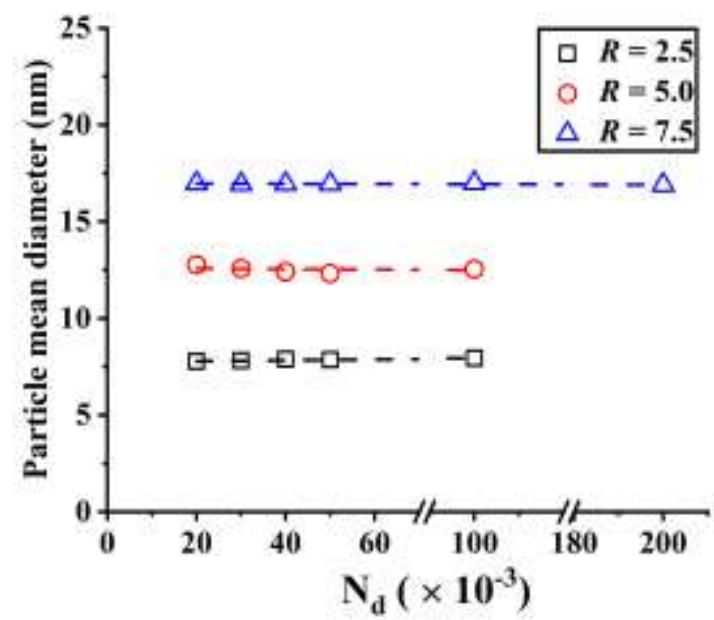

(a)

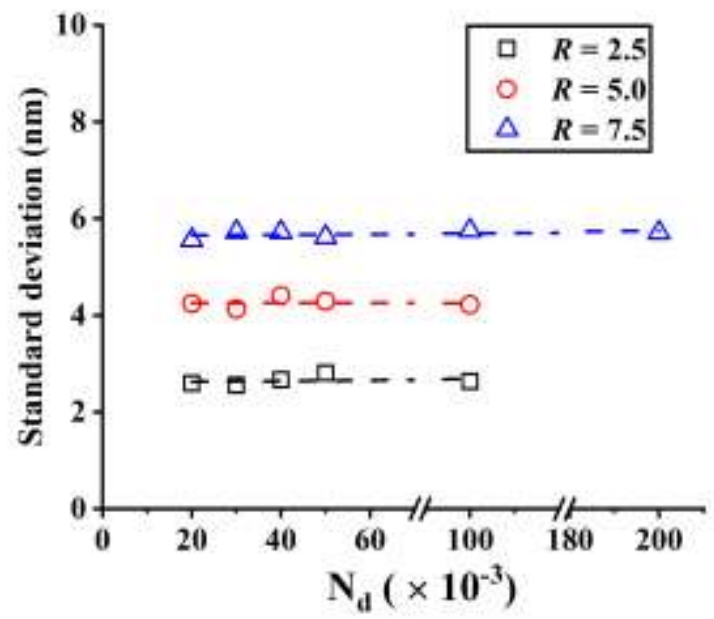

(b)

Figure S2. Effect of drop sample size on (a) particle mean diameter, (b) standard deviation. Dash line represents best fitted line. Note that the labels in $\mathrm{x}$-axis are given in the scale of $10^{-3}$. 
(SD) do not depend on the sample size, shown in Figures S2 (a) and (b) respectively. Finally, an optimized drop population of 50000 was chosen for the simulation.

\section{Different probable coagulation mechanisms:}

Various elementary events are involved in the formation of nanoparticles, via the waterin-oil microemulsion route, as shown in Figure S3. To this end, three different combinations of drop-coalescence and particle-coagulation schemes were evaluated (schemes S1, S2 and S3) and compared with experimental results (discussed in the main paper). Scheme S1 represents two drops devoid of particle, can coalesce to form a bigger drop containing all reagent and product molecules. Scheme S2 shows that, one drop containing particle can coalesce with another drop and form a bigger drop, containing the particle and other molecules.

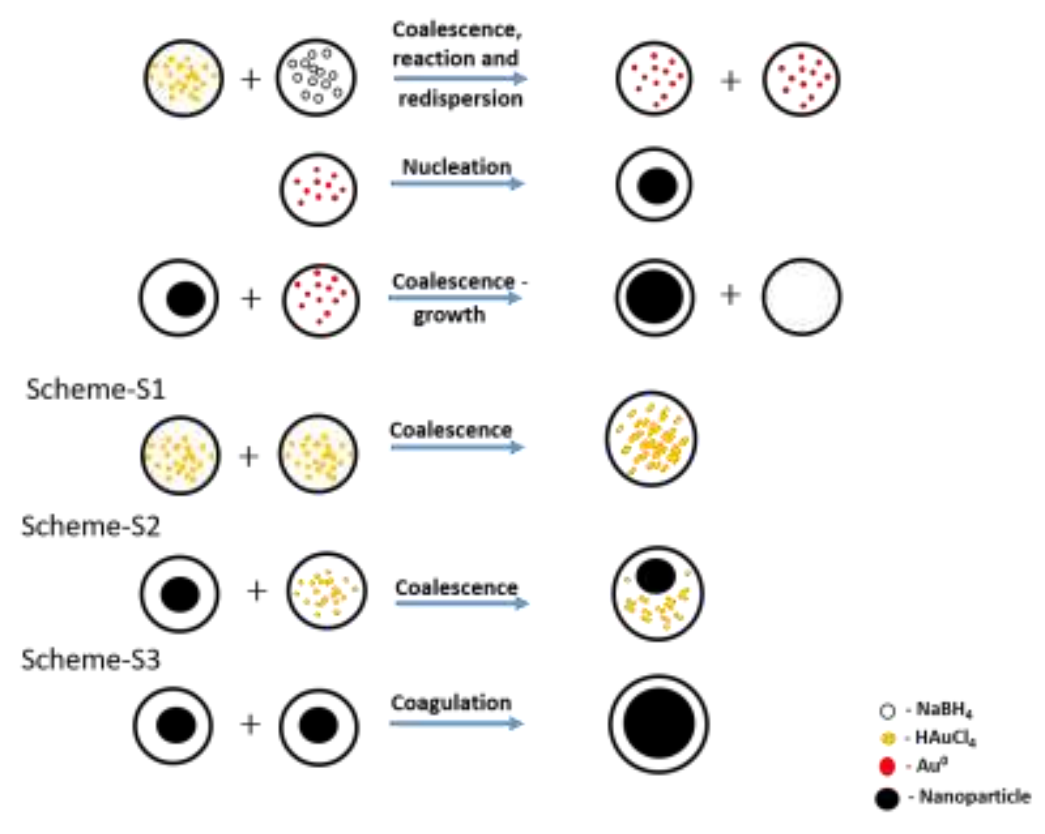

Figure S3. Possible elementary events involved in the formation of nanoparticles, in waterin-oil microemulsion route.

Finally, scheme S3 shows, only both drops containing particles are allowed to undergo coagulation and form a bigger drop containing a single bigger, coagulated particle. Since scheme S3 on its own gives the exact same result as that of schemes S1-S3 combined, or only schemes S2-S3 combined (Figure S4); hence, scheme S3 alone is the only necessary and 
sufficient mechanism for explaining the overall coagulation event, leading to the correct prediction of experimental trends and values.

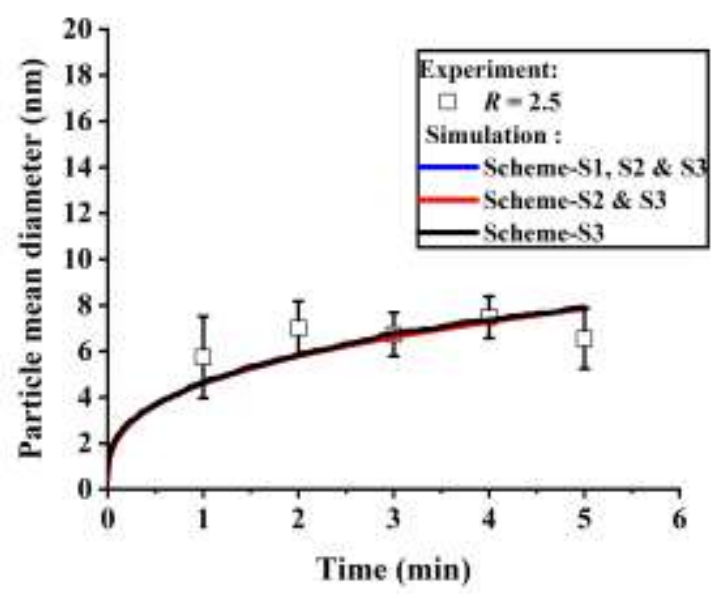

(a)

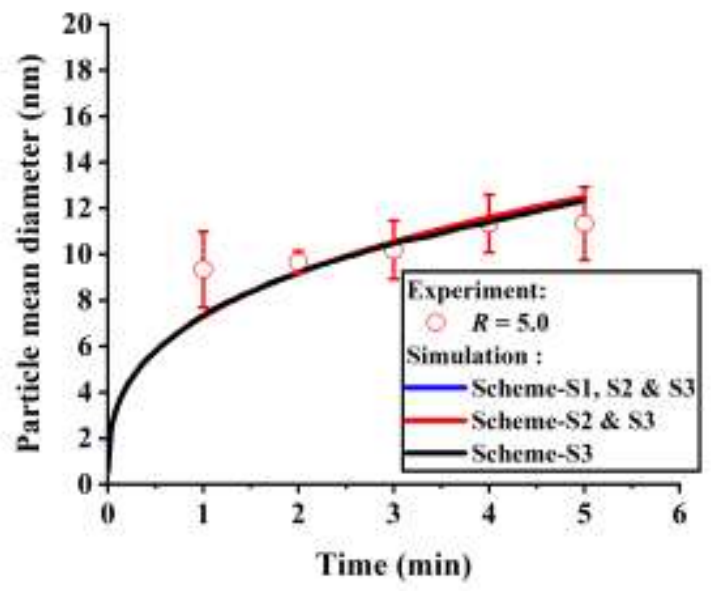

(b)

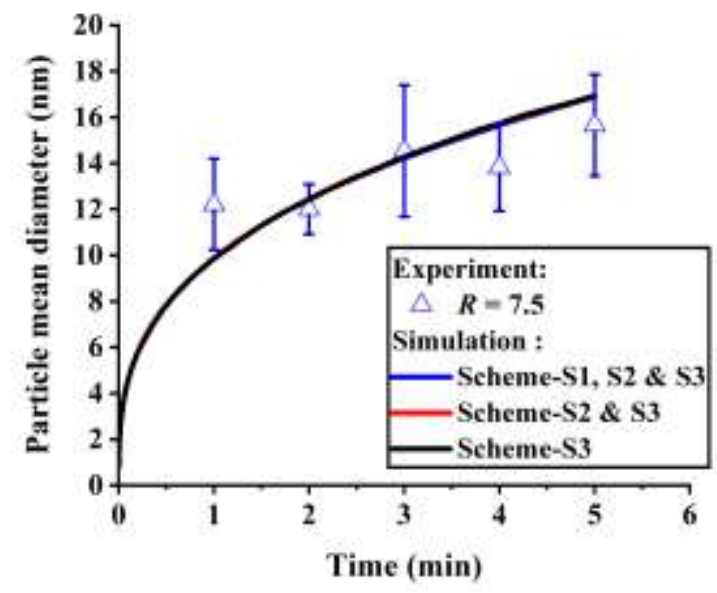

(c)

Figure S4. Comparison of experimental data (DLS) against different schemes of kMC simulation, for prediction of temporal evolution of GNPs diameter for different values of $R$. (a) $R=2.5$, (b) $R$ $=5.0$, and (c) $R=7.5$. Empty symbols, mean particle diameter from DLS; blue line, kMC with schemes S1, S2 and S3 combined; red line, kMC with schemes S2 and S3 combined; black line, $\mathrm{kMC}$ with only scheme S3. GNPs synthesized with $\left[\mathrm{HAuCl}_{4}\right]=1 \mathrm{mM},\left[\mathrm{NaBH}_{4}\right]=2 \mathrm{mM}$, and $[\mathrm{AOT}]=0.1 \mathrm{M}$. Error bars represents standard deviation from the mean of three individual experiments using DLS.

Based on the above conclusion, the extra steps in Figure S3 (schemes S1 and S2) are eliminated to arrive at the overall mechanism of nanoparticle formation in a microemulsion system, as depicted in Figure 1 of the main paper. 


\section{Comparison of particle size distribution from experiment and simulation:}

Final particle size distribution (PSD) of GNPs, obtained from TEM images were compared with the current kMC simulation (Figure S5).

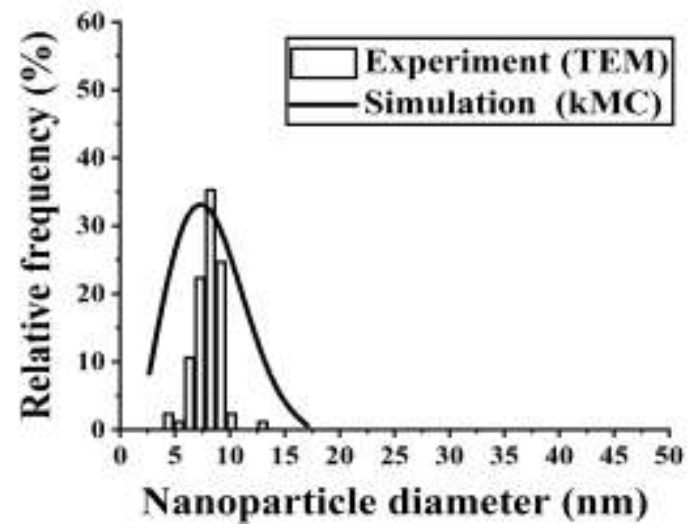

(a)

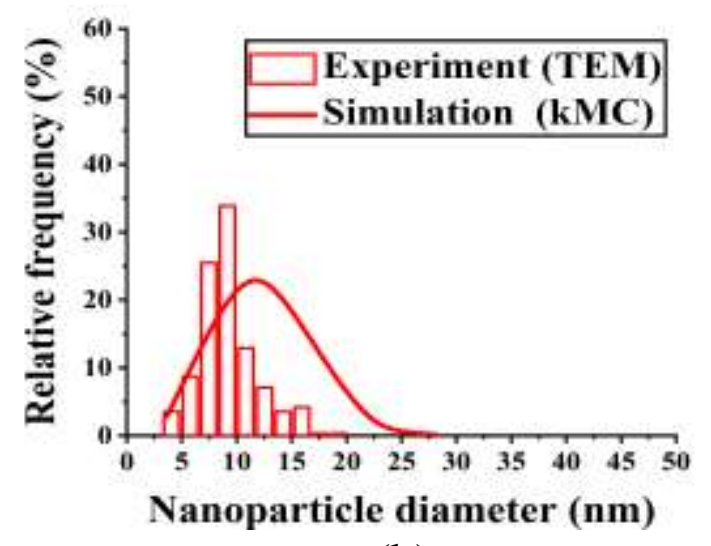

(b)

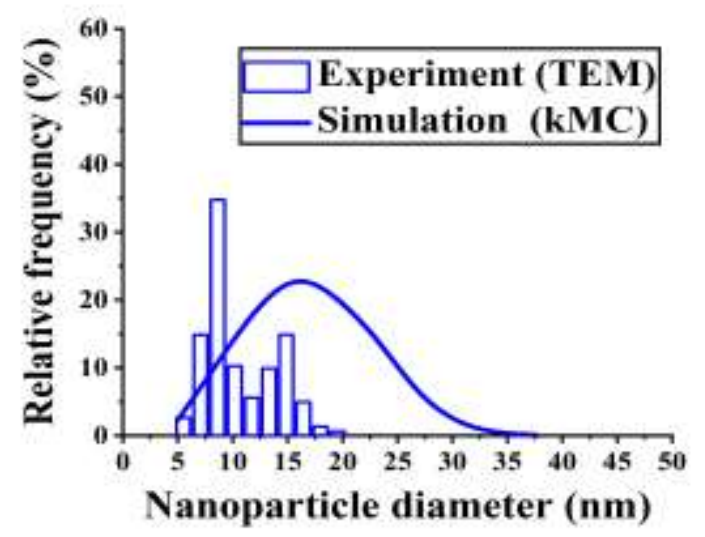

(c)

Figure S5. Comparison of PSD of GNPs from experiment (TEM) with current kMC simulation for different values of $R$ at $t=5 \mathrm{~min}$. (a) $R=2.5$, (b) $R=5.0$, and (c) $R=7.5$. Empty box, PSD from experiment and solid line, PSD from kMC simulation. GNPs synthesized with $\left[\mathrm{HAuCl}_{4}\right]=1 \mathrm{mM}$, $\left[\mathrm{NaBH}_{4}\right]=2 \mathrm{mM}$, and $[\mathrm{AOT}]=0.1 \mathrm{M}$. A total of 300 particles were randomly measured to obtain the PSD of GNPs using TEM.

Although current simulation does not match exactly with experimental results, but both experiment and simulated PSD shifts towards increasing size, with increasing $R$. As only 300 GNPs from TEM images could be measured, to obtain the PSD from a single individual experiment (corresponding to each value of $R$ ), so the TEM distribution may not represent the 
actual particle population very accurately. Therefore, we have used the PSD of DLS measurements for comparison with simulation in Figure 6 of the main paper.

In this regard, please note as the scattering coefficient of gold nanoparticle is at least of 5 orders higher compared to dye ${ }^{1}$ and water, ${ }^{2}$ the intensity of light scattered by AOT is therefore negligible. On similar line, the diameter of CdS nanoparticle measured by both UV spectrometer and DLS does not vary much. ${ }^{3}$ In fact diameter of CdS nanoparticle measured by DLS $(5 \mathrm{~nm})$ is $0.6 \mathrm{~nm}$ less compared to UV spectrometer $(5.6 \mathrm{~nm})$. This measurements confirm that AOT chain length $(2 \mathrm{lc} \sim 2.2 \mathrm{~nm})$ has no significant contribution to the hydrodynamic diameter of nanoparticles. On the other hand TEM represents only a subset population of 1 set of experiment, compared to DLS. On this basis we compared our simulation results with DLS data.

\section{Choice of nature of drop to be added after coagulation of two drops, each containing a particle:}

Case 1: A randomly chosen drop was added after each coagulation event (sometimes having a particle, and sometimes not).

Case 2: A drop containing particle was added after each coagulation event.

In case 1, the simulation results suffer, because the total number of particles in the system goes down monotonically, eventually making the simulation results statistically unreliable, due to only a small particle population in the system at the end of the simulation, which is equal to the experimental process time. In contrast, in case 2 , the total number of particles are preserved, till the end of the simulation. This results in the current proposed coagulation event (case 2) to be statistically reliable because of the large population of particles even towards the end of simulation. 
As an example, at $\mathrm{R}=7.5, \mathrm{t}=20 \mathrm{~s}$, for $\mathrm{N}_{\mathrm{d}}=50000$, for case 1 and 2 , only 20 and 6274 number of particles are left in the system. For such a small population (in case 1), the particles mean diameter fluctuates around the converged particle mean diameter (in case 2), shown in Figure S6. Therefore, it is clear that, the case 2 is more consistent and statistically reliable. Hence, we finalized case 2 (i.e. added drop always contains particle after each coagulation event) in our current proposed mechanism.

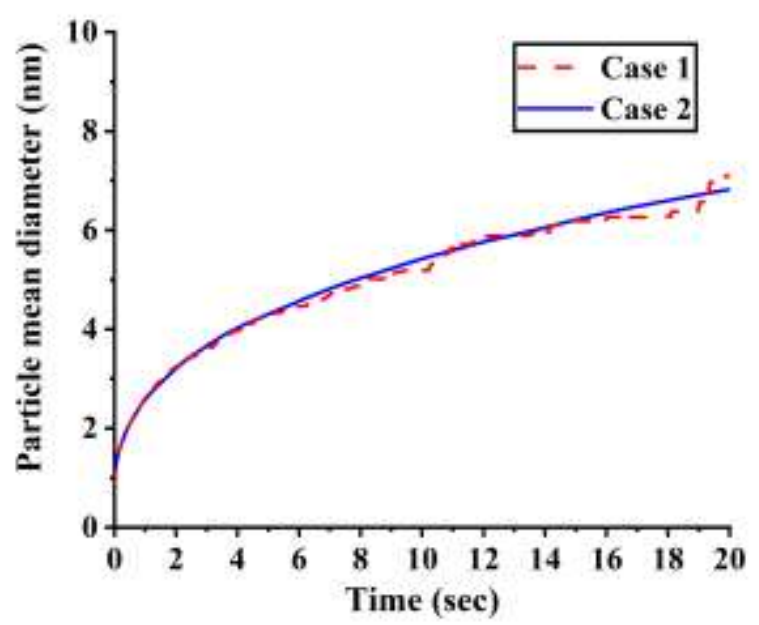

Figure S6. Comparison of particle mean diameter from case 1 and 2. At $R=7.5, \mathrm{~N}_{\mathrm{d}}=50000$. 


\section{REFERENCES:}

1. Jain, P. K.; Lee, K. S.; El-Sayed, I. H.; El-Sayed, M. A. Calculated absorption and scattering properties of gold nanoparticles of different size, shape, and composition: Applications in biological imaging and biomedicine. J. Phys. Chem. B 2006, 110, 7238 7248.

2. Jonasz, M.; Fournier, G.R. Light Scattering by Particles in Water: Theoretical and Experimental Foundations. Academic Press: San Diego, CA, USA, 2007, 145-265.

3. Ethayaraja, M.; Dutta, K.; Muthukumaran, D.; Bandyopadhyaya, R. Nanoparticle Formation in Water-in-Oil Microemulsions: Experiments, Mechanism, and Monte Carlo Simulation. Langmuir 2007, 23, 3418-3423. 